\title{
Uso de Antagonista de GnRH (Cetrorelix) em Dose Única para Evitar Ovulações Prematuras em Ciclos de Fertilização Assistida
}

\author{
Single Dose of GnRH Antagonist (Cetrorelix) to Avoid Premature \\ Ovulation in Assisted Fertilization Cycles
}

Pedro Luís Rosan, Gustavo Salata Romão, Rosana Maria dos Reis, Marcos Dias de Moura, Rui Alberto Ferriani

\section{RESUMO}

Objetivo: verificar a eficácia de uma dose única subcutânea de acetato de cetrorelix em evitar a ovulação prematura em ciclos de fertilização assistida.

Métodos: estudo prospectivo, randomizado e controlado, pelo qual foram avaliados 20 ciclos de estimulação ovariana em mulheres submetidas a fertilização assistida, 10 das quais utilizaram o esquema tradicional de bloqueio hipofisário com análogos de GnRH em doses diárias (grupo controle) e 10 utilizaram antagonista de $\mathrm{GnRH}$ em dose única de $3 \mathrm{mg}$ no $7^{\circ}$ dia de estimulação ovariana (grupo cetrorelix). Foram dosados $F S H, L H$, estradiol e progesterona no soro no primeiro e sétimo dia da estimulação, no dia da injeção de HCG e no dia da captação de oócitos. Os grupos foram comparados entre si quanto a eficácia do bloqueio hipofisário (nivel de progesterona no dia da aplicação do HCG) e desempenho nos ciclos de fertilização assistida (ampolas de gonadotrofinas utilizadas, folículos maiores que $18 \mathrm{~mm}$, oócitos captados, taxas de fertilização, implantação e gravidez) utilizando os testes de MannWhitney e exato de Fisher.

Resultados: não houve diferença significativa entre os grupos controle e cetrorelix, respectivamente, para a mediana da idade (31,5 e 34 anos), indice de massa corpórea (24 e 22), ampolas de gonadotrofinas utilizadas (34 e 32), foliculos recrutados (3,5 e 3,0), oócitos captados (11 e 5), embriões obtidos (4 e 3), taxas de fertilização (93,7 e 60\%, $p=0,07)$ e gravidez $(50$ e $60 \%, p=0,7)$. Em ambos os grupos observou-se bloqueio hipofisário eficaz durante o periodo de estimulação ovariana.

Conclusões: estes resultados confirmam a eficácia da dose única de $3 \mathrm{mg}$ de acetato de cetrorelix em prevenir ovulações prematuras em pacientes submetidas a fertilização assistida, mostrando tendência a obtenção de menor número de embriões e menores taxas de fertilização no grupo cetrorelix em relação ao grupo controle. As taxas de implantação e gravidez foram semelhantes entre os dois grupos. Estudos prospectivos com maior número de pacientes são necessários para confirmar estes achados.

PALAVRAS CHAVE: Antagonistas de GnRH. Acetato de cetrorelix. Fertilização assistida.

Setor de Reprodução Humana do Departamento de Ginecologia e Obstetrícia da Faculdade de Medicina de Ribeirão Preto da Universidade de São Paulo.

Correspondência:

Rui A Ferriani

Departamento Ginecologia e Obstetrícia da Faculdade de Medicina de Ribeirão Preto, USP

14049-900 - Ribeirão Preto - SP

e-mail: raferria@fmrp.usp.br

Agradecimentos à: Fundação de Amparo a Pesquisa do Estado de São Paulo (FAPESP) pelo auxilio financeiro e à "Asta Médica" pela cessão dos medicamentos utilizados neste estudo.
Introdução

A estimulação ovariana com gonadotrofinas, classicamente utilizada em ciclos de reprodução assistida, tem como desvantagem a possibilidade de ocorrência de pico prematuro de $\mathrm{LH}$, que pode comprometer o processo normal de maturação folicular, exercendo efeito deletério sobre a qua- 
lidade dos oócitos e levando ao cancelamento dos ciclos de estimulação ovariana ${ }^{1}$. Além disto, o aumento das concentrações de progesterona subseqüente ao pico prematuro de $\mathrm{LH}$ pode exercer efeito negativo sobre o endométrio, promovendo redução nas taxas de gravide $z^{2}$.

A supressão reversível da função pituitária com o uso de agonistas do GnRH tem melhorado a eficácia da terapia com gonadotrofinas, pois evita o pico prematuro de $\mathrm{LH}$, reduzindo a freqüência de luteinização prematura para aproximadamente $2 \%$ dos casos ${ }^{1-3}$.

Os agonistas do GnRH, após efeito inicial estimulatório sobre a hipófise (efeito flare-up), induzem a dessensibilização das células gonadotróficas pela redução no número de receptores de GnRH na membrana celular ${ }^{1}$ (efeito downregulation). O protocolo clássico de bloqueio hipofisário com agonistas de GnRH, chamado de protocolo longo, apresenta algumas desvantagens, dentre as quais merecem destaque a possibilidade de longo efeito flare-up levando a período de tratamento relativamente longo (em torno de 14 dias) antes que a supressão da liberação de gonadotrofinas endógenas ocorra e que a estimulação com gonadotrofinas exógenas (gonadotrofinas de mulher menopausada - HMG e/ou hormônio folículo-estimulante recombinante - recFSH) possa ser inici$\mathrm{ada}^{4}$; a dessensibilização da glândula pituitária leva à ocorrência de sintomas decorrentes da supressão de estrogênios, como fogachos e secura vaginal ${ }^{5}$; o aumento do risco de hiperestimulação ovariana, especialmente em casos de aplicação de protocolo de estimulação ovariano curto ${ }^{6}$; a necessidade do uso de doses elevadas de gonadotrofinas exógenas para a maturação folicular ${ }^{7}$; maior tempo necessário para a recuperação da secreção endógena de gonadotrofinas devido ao efeito downregulation sobre os receptores hipofisário de GnRH e vesículas de armazenamento gonadotróficas ${ }^{8}$.

Com relação aos antagonistas do $\mathrm{GnRH}$, os primeiros compostos sintetizados apresentavam como inconveniente a alta incidência de reações alérgicas durante o seu uso ${ }^{9}$. Os antagonistas atualmente disponiveis para uso clínico apresentam alta potência biológica associada a reduzida atividade liberadora de histamina, sendo o mais extensivamente estudado, o acetato de cetrorelix ${ }^{7,10}$. O uso dos antagonistas do GnRH promove bloqueio competitivo dos receptores de GnRH, que leva a imediata supressão da secreção de gonadotrofinas ${ }^{11}$. Desta forma, a duração do tratamento e o número de ampolas necessárias para a estimulação ovariana controlada pode ser menor ${ }^{1,10,12}$. Quando administrados durante a fase folicular, os antagonistas do GnRH podem evitar picos prematuros de $\mathrm{LH}$ ou mesmo interromper a sua elevação durante o período de estimulação ovariana controlada. Além disto, a recuperação da função da pituitária ocorre mais rapidamente após uso dos antagonistas do que em relação aos agonistas do $\mathrm{GnRH}^{8}$.

Vários protocolos de estimulação ovariana utilizando antagonistas do GnRH têm sido descritos na literatura ${ }^{5}$. O regime de múltiplas doses, único disponivel no Brasil, requer injeções diárias com baixa dosagem de antagonistas de GnRH (cetrorelix), iniciadas no $5^{\circ}$ ou $6^{\circ}$ dia da estimulação ovariana até o dia da injeção de hCG. Já o regime de dose única requer injeção com maior dosagem de antagonista do GnRH (cetrorelix) em torno do $7^{\circ}$ dia da estimulação ovariana ${ }^{5}$.

Este estudo prospectivo controlado tem como objetivos verificar se a administração de uma única dose subcutânea de $3 \mathrm{mg}$ de cetrorelix inibe a ovulação prematura em pacientes submetidas a estimulação ovariana controlada para técnicas de reprodução assistida, além de avaliar o desempenho das pacientes submetidas a este protocolo em relação ao protocolo clássico de estimulação ovariana com agonistas de GnRH. Tal regime constitui uma primeira experiência brasileira com dose única.

\section{Pacientes e Métodos}

Este estudo prospectivo controlado e randomizado foi desenvolvido no Setor de Reprodução Humana do Hospital das Clínicas de Ribeirão Preto (HCFMRP-USP) durante o ano de 2002, sendo o seu protocolo avaliado e aprovado pelo Comitê de Ética em Pesquisa local.

Foram selecionadas mulheres acompanhadas no Ambulatório de Infertilidade Conjugal do HCFMRP-USP que apresentavam indicações para procedimentos de fertilização assistida (fertilização in vitro - FIV ou injeção intracitoplasmática de espermatozóides - ICSI)

Todas as pacientes selecionadas haviam sido submetidas previamente ao protocolo básico de investigação dos casais inférteis do HCFMRP-USP, que inclui exame ginecológico, ecografia pélvica transvaginal, histeroscopia e laparoscopia diagnósticas, dosagem de FSH no $3^{\circ}$ dia do ciclo menstrual e avaliação do espermograma do parceiro.

Uma vez selecionadas, as pacientes foram devidamente informadas sobre os objetivos e protocolos do estudo e, estando de acordo com o mesmo, assinaram o termo de consentimento pós-informação. 
Foram consideradas elegiveis para este estudo as pacientes com idade entre 18 e 39 anos, cuja investigação básica foi compatível com útero normal e dois ovários funcionantes e que apresentavam indicações clássicas para fertilização assistida, como fator masculino grave, obstrução tubária bilateral ou falhas em tentativas prévias de reprodução assistida com técnicas de baixa complexidade.

Foram excluídas deste estudo as pacientes que apresentavam duração do ciclo menstrual menor que 24 dias ou maior que 35 dias, elevada variabilidade na duração do ciclo menstrual (mais do que \pm 3 dias), história prévia de reação alérgica a cetrorelix ou hormônios peptídeos sintéticos e contra-indicações para o uso de gonadotrofinas. Excluímos as pacientes que realizaram testes funcionais hormonais ou usaram contraceptivos hormonais injetáveis nos seis últimos meses, as que foram submetidas a quatro ou mais tentativas anteriores de fertilização assistida e as que apresentavam história de má resposta a estimulação ovariana com hMG/FSH em ciclos anteriores. Foram também excluídas as que apresentassem síndrome de ovários policísticos (definido pela elevação dos niveis de LH, relação LH/ FSH $>2$, testosterona elevada e aparência ultrasonográfica típica de ovários policísticos), baixa reserva ovariana $\left(\mathrm{FSH}>10 \mathrm{UI} / \mathrm{L}\right.$ no $3^{\circ}$ dia do ciclo), insuficiência do corpo lúteo, função ovariana diminuída, endometriose grave (classe III ou IV), miomas uterinos ou formação cística ovariana com mais de $2 \mathrm{~cm}$ de diâmetro antes do início da estimulação.

As pacientes foram distribuidas de forma randomizada em dois grupos com 10 mulheres, sendo o primeiro grupo (grupo controle) submetido à estimulação ovariana padronizada no serviço, que utiliza agonistas de GnRH (acetato de leuprolida) em protocolo longo, e o segundo grupo (grupo cetrorelix) submetido ao mesmo esquema, exceto pelo uso de antagonistas de GnRH (cetrorelix) ao invés de agonistas do GnRH.

Em resumo, as pacientes do grupo controle foram submetidas a uma injeção de $0,5 \mathrm{mg}$ de acetato de leuprolida a partir do $10^{\circ}$ dia anterior ao dia basal (primeiro dia da estimulação ovariana), quando foi realizada avaliação inicial dos ovários com ultra-sonografia. A partir do dia basal iniciou-se a estimulação ovariana controlada com 3 a 4 ampolas diárias de HMG ou FSH. No sétimo dia de estimulação as pacientes retornaram para nova avaliação ultra-sonográfica e reajuste das doses de gonadotrofinas em função do crescimento folicular observado. Por ocasião da maturidade folicular (pelo menos um folículo com diâmetro médio de $18 \mathrm{~mm}$ ), a administração de gonadotrofinas e análogos de GnRH foi suspensa, procedendo-se à administração de 10.000 UI de HCG. A captação de oócitos foi realizada 35 horas após a injeção de HCG. Após 72 horas em relação à captação, realizou-se a transferência embrionária em número máximo de seis embriões. A reposição da fase lútea foi realizada com progesterona natural micronizada (Utrogestan ${ }^{\circledR}$ ) $600 \mathrm{mg}$ ao dia, dividida em 2 tomadas a partir da captação de oócitos até o $14^{\circ}$ dia pós-transferência, quando foi realizado o teste gravídico urinário visual e, caso a paciente estivesse grávida, a suplementação progestínica seria mantida até a $12^{\text {a }}$ semana de gestação.

No grupo do cetrorelix uma única dose de 3 mg de acetato de cetrorelix (doado pela Asta-Médica AG, Frankfurt, Germany) foi administrada por via subcutânea na parede abdominal abaixo da cicatriz umbilical no $7^{\circ}$ dia de estimulação ovariana. Caso o crescimento folicular observado nos quatro dias subseqüentes à injeção de cetrorelix não permitisse a indução da ovulação com HCG, doses adicionais diárias de 0,25 mg de cetrorelix eram administradas por via subcutânea até o dia da maturidade folicular.

Em ambos os grupos foram colhidas amostras do sangue periférico das pacientes no primeiro e no sétimo dia da estimulação ovariana, no dia da injeção do HCG e no dia da captação de oócitos. Eram retirados $8 \mathrm{~mL}$ de sangue venoso por meio do BD Vacutainer SST. Após a coagulação, este era centrifugado a $2500 \mathrm{rpm}$ durante $10 \mathrm{mi}-$ nutos. O sobrenadante (soro) era armazenado em tubos de ensaio e colocado no congelador a uma temperatura de $-20^{\circ} \mathrm{C}$ para posterior dosagem.

Em cada amostra de primeiro dia foram dosados FSH, LH, estradiol, progesterona e o $\beta$ HCG e em cada amostra do sétimo dia, do dia do HCG e do dia da captação oocitária foram dosados estradiol e progesterona. As dosagens hormonais nas amostras foram realizadas por meio do método enzimático de imunoquimioluminescência utilizando o aparelho Immulite DPC - Med Lab, sendo um mesmo kit empregado para a dosagem de cada hormônio específico (Immulite ${ }^{\circledR}$ Progesterone, Immulite ${ }^{\circledR}$ Estradiol, Immulite ${ }^{\circledR}$ FSH e Immulite ${ }^{\circledR}$ LH, todos da Diagnostic Products Corporation; Los Angeles, USA).

Além das dosagens hormonais supracitadas, foram avaliados diversos parâmetros relacionados ao ciclo de estimulação ovariana controlada, tais como o número e tamanho dos folículos no dia da administração de hCG, o número de oócitos (puncionados e captados) e embriões transferiveis, o perfil de estradiol, a dose e duração do tratamento de estimulação ovariana, a taxa de fertilização, a 
taxa de implantação embrionária, a taxa de gravidez bioquímica ( $\beta \mathrm{HCG}$ positivo), a ocorrência de sindrome do hiperestímulo ovariano e a tolerabilidade da droga estudada (cetrorelix).

A distribuição das causas e da classificação da infertilidade também foi similar entre os dois grupos. O grupo controle foi composto de $90 \%$ de pacientes com fator masculino como causa de infertilidade, $10 \%$ de fator tubário, ao passo que o grupo do cetrorelix foi composto por $80 \%$ de pacientes com fator masculino, $10 \%$ de fator tubário e $10 \%$ de esterilidade sem causa aparente. Com relação à classificação da infertilidade, foi observado $80 \%$ de infertilidade primária no grupo controle e $90 \%$ no grupo do cetrorelix.

A análise comparativa entre os grupos foi realizada pelo teste de Mann-Whitney para variáveis quantitativas e do teste exato de Fisher para comparações entre as taxas, utilizando-se o programa GraphPad Prisma, com nível de significância de $5 \%$. Os dados são apresentados por sua mediana e pelo $1^{\circ}$ e $3^{\circ}$ quartil.

Todas as pacientes apresentaram $\beta H C G$ sérico com nivel indetectável na triagem hormonal inicial no $1^{\circ}$ dia da estimulação ovariana (dia do basal). Nenhuma diferença entre os dois grupos foi encontrada, com relação a idade $(31,5$; $28,2-$ 32,7 no grupo controle e $34 ; 30,2-35$ no grupo do cetrorelix), indice de massa corporal (IMC) $(24,6$; $21,5-25,7$ no grupo controle e $22 ; 20,3-26,5$ no gru- po do cetrorelix) e nível sérico do FSH no $3^{\circ}$ dia do ciclo menstrual $(4,7 ; 3,5-6,4$ no grupo controle e 4,$1 ; 3,4-6,4$ no grupo do cetrorelix).

\section{Resultados}

Das pacientes que receberam cetrorelix em dose única de $3 \mathrm{mg}(\mathrm{n}=10)$, apenas uma paciente recebeu uma dose adicional de $0,25 \mathrm{mg}$ de cetrorelix devido a imaturidade folicular no quarto dia após a dose inicial. Neste caso, a maturidade folicular ocorreu no quinto dia após a dose inicial de cetrorelix, ocasião em que foi administrado HCG.

Em nenhuma das pacientes $(n=20)$ foram observados indícios de ovulação precoce (progesterona sérica maior que $1,00 \mathrm{ng} / \mathrm{mL}$ ) ou síndrome do hiperestímulo ovariano.

Com relação à tolerabilidade do cetrorelix, duas pacientes apresentaram mácula eritematosa não pruriginosa no local da injeção, a qual desapareceu em uma hora, não havendo necessidade de qualquer tratamento adicional.

A duração em dias foi de 9,$0 ; 8,0-10,7$ para o grupo controle e de 9,$0 ; 8,0-9,7$ para o grupo do cetrorelix e o número de ampolas de HMG utilizadas durante o período de estimulação foi 34,0; 32,041,5 para o grupo controle e de 32,0; 30,0-36,5 para o grupo do cetrorelix, sendo, assim, semelhantes em ambos os grupos, conforme mostra a Tabela 1.

Tabela 1 - Comparação entre o uso de agonistas (controle) e o uso de antagonistas de GnRH (cetrolix) na estimulação ovariana para fertilização assistida, representando-se respectivamente a mediana, o primeiro e o terceiro quartis.

Variáveis

Controle

Mediana $\left(1^{\circ}-3^{\circ}\right.$ quartil)

Idade (anos)

IMC

$31,50(28,25-32,75)$

$\mathrm{FSH}$ do $3^{\circ}$ dia

$24,63(21,50-25,75)$

Número de dias de estimulação

$4,70(3,50-6,45)$

Número de ampolas de HMG

$9,00(8,00-10,75)$

Número de folículos de 11 a $17 \mathrm{~mm}$

$34,00(32,00-41,50)$

$14,00(6,25-16,50)$

Número de folículos $>18 \mathrm{~mm}$

Número de oócitos puncionados

$3,50(2,25-5,50)$

Número de oócitos captados

Taxa de fertilização (\%)

$17,00(11,50-23,75)$

$11,00(7,25-11,75)$

$93,75 \%$

Número de embriões

Taxa de implantação (\%)

$4,00(3,00-4,75)$

$12,5 \%$

$50 \%$

\section{Tratamento}

\section{Cetrolix}

Mediana $\left(1^{\circ}-3^{\circ}\right.$ quartil)

$\begin{array}{cc}34,00(30,25-35,00) & 0,2475^{(1)} \\ 22,03(20,34-26,53) & 0,3150^{(1)} \\ 4,15(3,47-6,47) & 0,9118^{(1)} \\ 9,00(8,00-9,75) & 0,5787^{(1)} \\ 32,00(30,00-36,50) & 0,2799^{(1)} \\ 6,50(4,25-9,25) & 0,1051^{(1)} \\ 3,00(2,00-4,00) & 0,7394^{(1)} \\ 11,00(7,25-11,75) & 0,1431^{(1)} \\ 5,00(4,00-7,25) & 0,054^{(1)} \\ 60 \% & 0,0753^{(2)} \\ 3,00(2,00-4,75) & 0,2799^{(1)} \\ 33,33 \% & 0,6842^{(2)} \\ 60 \% & 0,7394^{(2)}\end{array}$

${ }^{1}$ teste de Mann-Whitney; ${ }^{2}$ teste exato de Fisher.

IMC = índice de massa corporal 
No grupo controle, os níveis de progesterona em $\mathrm{ng} / \mathrm{mL}$ foram 0,$5 ; 0,4-0,6$ no sétimo dia de estimulação e 0,8; 0,6-0,9 no dia da injeção do HCG. No grupo do cetrorelix os niveis de progesterona em $\mathrm{ng} / \mathrm{mL}$ no sétimo dia de estimulação foram $(0,6 ; 0,5-0,7)$ e no dia da injeção do HCG, 0,6;0,40,8 . Com relação ao estradiol, no grupo controle os niveis em $\mathrm{pg} / \mathrm{mL}$ no dia da injeção do HCG foram 1815; 1097-3967 e no dia da captação de oócito, $842 ;$ 510-2262). No grupo do cetrorelix os niveis de estradiol em $\mathrm{pg} / \mathrm{mL}$ no dia da injeção do HCG foram 1179; 937-1516 e no dia da captação de oócito, 652; 553-955, não se observando diferença estatística significante entre os dois grupos (Figuras 1 e 2).

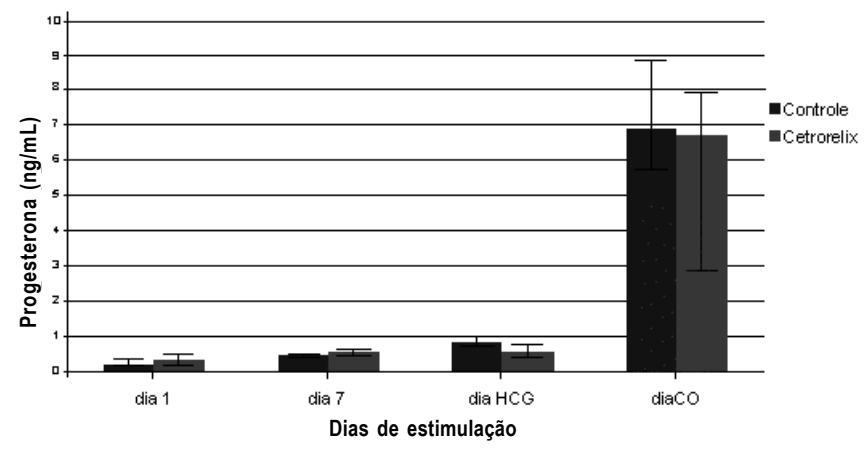

Figura 1 - Nível sérico de progesterona (Md; 1QT e 3QT) nos dois grupos de pacientes.

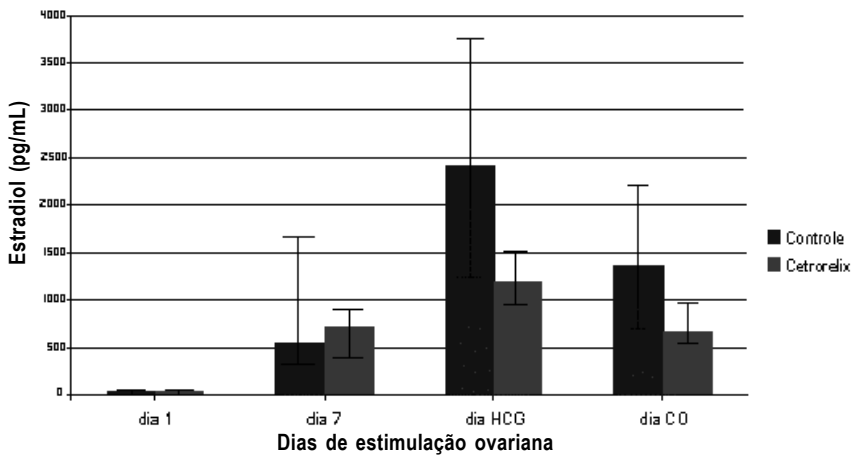

Figura 2 - Nivel sérico de estradiol (Md; 1QT e 3QT) nos dois grupos de pacientes.

Não houve diferença quanto ao número de folículos com diâmetro médio maior que $18 \mathrm{~mm}$ no dia da injeção de HCG $(3,5 ; 2,2-5,5$ para o grupo controle e 3,0; 2,0-4,0 para o grupo do cetrorelix) ou quanto ao número de folículos entre 11 e 17 $\mathrm{mm}$ (Tabela 1). O número total de oócitos puncionados $(17,0 ; 11,5-23,7$ grupo controle e 11,0; $7,2-11,7$ no grupo cetrorelix) e captados $(11,0 ; 7,2-$ 11,7 grupo controle e 5,$0 ; 4,0-7,2$ grupo cetrorelix) foi significativamente maior no grupo controle em relação ao grupo do cetrorelix. Não foram observadas diferenças significativas quanto às taxas de fertilização, ao número de embriões obtidos $(4,0$; $3,0-4,7$ no grupo controle e 3,0; $2,0-4,7$ no grupo cetrorelix), às taxas de implantação e às taxas de gravidez bioquímica, representadas pelo número de testes $\beta \mathrm{HCG}$ urinários positivos no $14^{\circ}$ dia póstransferência em relação ao número de transferências embrionárias realizadas (Tabela 1).

\section{Discussão}

Este estudo confirma a eficácia do cetrorelix na dose única de $3 \mathrm{mg}$ para inibir a ovulação precoce nos ciclos de estimulação ovariana controlada, em conformidade com achados de outros autores ${ }^{1,3,9,10}$. Nenhuma paciente apresentou nivel sérico de progesterona superior a $1 \mathrm{ng} / \mathrm{mL}$ no $7^{\circ}$ dia da estimulação ou no dia da injeção de HCG, sendo o nivel sérico de progesterona mais elevado detectado no grupo do cetrorelix de $0,9 \mathrm{ng} / \mathrm{mL}$ no $7^{\circ}$ dia da estimulação, antes da injeção do cetrorelix. Alguns estudos apontam para um índice de $2 \%$ de ovulação precoce nas pacientes em uso de agonistas de GnRH em ciclos de fertilização assistida ${ }^{1,3,5}$, embora não tenha sido observado nenhum caso de escape ovulatório nas pacientes do grupo controle deste estudo.

A tolerância ao cetrorelix foi considerada adequada, uma vez que apenas duas pacientes apresentaram mácula eritematosa não pruriginosa no local da injeção, a qual desapareceu sem qualquer tratamento em aproximadamente uma hora. O estudo de Olivennes et al. ${ }^{5}$ observou $15 \%$ de reação eritematosa passageira no local da injeção do cetrorelix.

Neste protocolo de dose única do antagonista do GnRH tivemos um caso onde foi necessária a administração de uma dose adicional de $0,25 \mathrm{mg}$ de cetrorelix no $5^{\circ}$ dia após a administração da dose única, sendo administrado neste mesmo dia o HCG. Em conformidade com nossos achados, Olivennes et al. ${ }^{5}$, em seu estudo com 115 mulheres em uso de cetrorelix ( $3 \mathrm{mg}$ dose única), relatam que 11 pacientes $(9,6 \%)$ necessitaram de uma dose adicional de 0,25 mg de cetrorelix. Uma análise de dois grandes estudos experimentais multicêntricos de fase IIIb confirmou a segurança e a eficácia do uso de antagonistas de GnRH em dose única de $3 \mathrm{mg}$ na prevenção de picos prematuros de $\mathrm{LH}^{13}$.

Não houve diferença na duração do período de estimulação ovariana e no número de ampolas de gonadotrofinas utilizadas entre os grupos, embora se possa observar tendência a redução do nú- 
mero de ampolas utilizadas no grupo do cetrorelix. Estudos com maior número de casos, nos quais se comparou o uso do antagonista com o uso de agonista de GnRH durante os ciclos de reprodução assistida, obtiveram como resultado menor número de dias e ampolas gastas durante a estimulação ovariana ${ }^{1,3,5,8,14}$. Explicação plausivel para este fato seria que até o $7^{\circ}$ dia de estimulação ovariana (quando geralmente se faz a administração dos antagonistas de $\mathrm{GnRH}$ ) haveria somatório de efeitos das gonadotrofinas endógenas e exógenas sobre a maturação folicular, e portanto a quantidade total de gonadotrofinas exógenas necessárias para a estimulação ovariana seria reduzida, bem como a duração deste período ${ }^{3}$. Por outro lado, no protocolo longo de estimulação ovariana com agonistas do GnRH, a secreção hipofisária de gonadotrofinas endógenas estaria completamente bloqueada na ocasião do início da estimulação ovariana, havendo necessidade de maior período de estimulação com maior quantidade de gonadotrofinas exógenas ${ }^{3}$.

Analisando os resultados da estimulação ovariana neste estudo, observamos tendência para a redução do número de folículos obtidos no dia de administração do HCG no grupo cetrorelix em relação ao grupo controle, embora sem significância estatística. É possivel que esta tendência seja ocasionada pela menor exposição às gonadotrofinas durante a estimulação ovariana no grupo cetrorelix em relação ao grupo controle. Este fato também poderia justificar a tendência ao menor número de oócitos captados e embriões obtidos no grupo do cetrorelix quando comparado ao grupo controle.

Outros estudos relataram número menor de folículos recrutados e de embriões obtidos quando se utilizam antagonistas de GnRH em relação aos protocolos tradicionais de estimulação com agonistas de $\mathrm{GnRH}^{5,8}$. Em recente metanálise, cinco estudos randomizados e controlados foram analisados para comparação entre os resultados da estimulação ovariana com antagonistas e análogos de GnRH (protocolo longo), em um dos quais se utilizou a dose única de $3 \mathrm{mg}$ de cetrorelix. Como conclusão desta metanálise os autores consideraram a utilização de antagonistas de GnRH em dose única um protocolo simples e factivel, mas com taxas inferiores de fertilização e número semelhante de picos prematuros de $\mathrm{LH}$ e de casos de sindrome da hiperestimulação grave em relação ao protocolo longo de estimulação com agonistas. Por isto o uso de antagonista do GnRH requer o esclarecimento adequado dos casais subférteis antes de recomendar o uso de antagonista do GnRH em lugar dos agonista ${ }^{14}$.

Os niveis séricos de estradiol no dia da injeção do HCG foram semelhantes entre ambos os grupos, com tendência a valores inferiores no gru- po do cetrorelix. Possivel explicação seria o fato de este grupo apresentar menor número de folículos produtores de estradiol.

Não foram observados casos da síndrome da hiperestimulação ovariana. Olivennes et $a .^{5}$, em 2000, referem menor incidência da sindrome da hiperestimulação ovariana em pacientes que fizeram uso de antagonista do GnRH. A análise de outros estudos ${ }^{14}$ não confirmou estes achados.

O número de embriões e as taxas de implantação e gravidez química foram semelhantes em ambos os grupos. Alguns estudos observaram menor número de embriões obtidos e menores taxas de implantação e gravidez quando se utilizam antagonistas de GnRH em relação ao uso de agonistas de GnRH ${ }^{14}$. Esta observação pode despertar questões relativas ao impacto do uso de antagonistas do GnRH sobre o endométrio e subseqüentemente sobre as taxas de implantação, bem como poderá confirmar a hipótese de que os antagonistas do GnRH interagem com o processo mitótico das células envolvidas na foliculogênese, repercutindo na qualidade da formação dos blastômeros ${ }^{15}$. Outra explicação possivel para uma tendência a menor taxa de implantação e gravidez é o fato de o grupo tratado com antagonistas produzir menor número de folículos e embriões ${ }^{5}$.

Alguns estudos se propuseram a avaliar a influência do peso corpóreo sobre os resultados da estimulação ovariana com antagonistas de GnRH para fertilização in vitro. A maioria destes estudos sugere uma correlação positiva entre o peso corpóreo e o número de folículos recrutados, mas não foi estabelecida nenhuma relação com as taxas de gravide $z^{16}$. Recentes revisões apontam para taxas de sucesso equivalentes quando se compara o uso de análogos com o uso de antagonistas de GnRH em pacientes normo-responsivas ${ }^{17}$.

Em resumo, este estudo confirmou a eficácia de inibição da ovulação precoce, bem como a boa tolerabilidade, segurança e a facilidade do uso do cetrorelix $3 \mathrm{mg}$ em dose única, administrado na fase folicular tardia durante os programas de fertilização assistida. Este protocolo mostrou tendência à utilização de menor número de ampolas de HMG para estimulação ovariana quando se utilizam antagonistas de GnRH, embora sem significância estatística. Da mesma forma houve tendência à obtenção de menor número de folículos captados, menor número de embriões, menor nível sérico de estradiol no dia do HCG e menor taxa de fertilização no grupo tratado com antagonistas de GnRH em relação ao grupo controle. As taxas de implantação e gravidez foram semelhantes entre os dois grupos. Estudos prospectivos com maiores casuísticas são necessários para a confirmação destes achados. 


\section{ABSTRACT}

Purpose: to verify the efficacy of a single subcutaneous dose of cetrorelix acetate to avoid premature ovulation in assisted fertilization cycles.

Methods: this is a prospective, controlled and randomized study, with 20 women undergoing ovarian stimulation for assisted fertilization, 10 of whom were submitted to classical GnRH agonist protocol (control group) while 10 utilized a 3-mg subcutaneous dose of the GnRH antagonist on the 7th day of ovarian stimulation (cetrorelix group). Serum FSH, $\mathrm{LH}$, estradiol and progesterone concentrations were assessed on the first, seventh, HCG administration and oocyte retrieval days. Both groups were compared for pituitary suppression (progesterone concentration on HCG day) and assisted fertilization cycle performance (gonadotropin ampoules utilized, follicles over $18 \mathrm{~mm}$, retrieved oocytes, fertilization, implantation and pregnancy rates), utilizing Mann-Whitney and Fisher exact tests.

Results: no significant difference was observed between control and cetrorelix groups, respectively, for medians of age (31.5 and 34 years), body mass index (24 and 22), gonadotropin ampoles utilized (34 and 32), follicles over 18 $\mathrm{mm}$ (3.5 and 3.3), retrieved oocytes (11 and 5), obtained embryos (4 and 3), fertilization (93,7 and 60\%, $p=0.07)$ and pregnancy rates (50 and $60 \%, p=0.07$ ). Efficient pituitary blockade through the ovarian stimulation period was observed for both groups.

Conclusions: these results confirm the efficacy of a 3-mg dose of cetrorelix acetate to prevent premature ovulation in patients undergoing assisted fertilization and suggest a tendency towards a smaller number of embryos and fertilization rates in the cetrorelix group than in the control group. Implantation and pregnancy rates were similar between groups. Other prospective studies with a greater number of patients should be performed to confirm these results.

KEYWORDS: GnRH antagonists. Cetrorelix acetate. Assisted fertilization.

\section{Referências}

1.Diedrich K, Diedrich C, Santos E, et al. Suppression of the endogenous luteinizing hormone surge by the gonadotrophin-releasing hormone antagonist Cetrorelix during ovarian stimulation. Hum Reprod 1994; 9:788-91.

2. Reissmann T, Schally AV, Bouchard P, Riethmiiller $\mathrm{H}$, Engel J. The LHRH antagonist cetrorelix: a review. Hum Reprod Update 2000; 6:322-31.

3. Albano C, Smitz J, Camus M, et al. Hormonal profile during the follicular phase in cycles stimulated with a combination of human menopausal gonadotrophin and gonadotrophin-releasing hormone antagonist (Cetrorelix). Hum Reprod 1996; 11:2114-8.

4. Filicori M, Cognigni GE, Arnone $\mathrm{R}$, et al. Role of different $\mathrm{GnRH}$ agonist regimens in pituitary suppression and the outcome of controlled ovarian hyperstimulation. Hum Reprod 1996; 11 (Suppl 3):123-32.
5. Olivennes F, Belaisch-Allart J, Emperaire JC, et al. Prospective, randomized, controlled study of in vitro fertilization-embryo transfer with a single dose of a luteinizing hormone-releasing hormone (LH-RH) antagonist (cetrorelix) or a depot formula of an LH$\mathrm{RH}$ agonist (triptorelin). Fertil Steril 2000; 73:314-20.

6. Ron-El R, Raziel A, Schachter M, Strassburger D, Kasterstein E, Friedler S. Induction of ovulation after GnRH antagonists. Hum Reprod Update 2000; 6:318-21.

7. Devroey P. GnRH antagonists. Fertil Steril 2000; 73:15-7.

8. European and Middle East Orgalutran Study Group. Comparable clinical outcome using the GnRH antagonist ganirelix or a long protocol of the GnRH agonist triptorelin for the prevention of premature LH surges in women undergoing ovarian stimulation. Hum Reprod 2001; 16:644-51.

9. Schmidt F, Sundaram K, Thau RB, Bardin CW. [AcD-NAL(2) 1,4FD-Phe2,D-Arg6]-LHRH, a potent antagonist of LHRH, produces transient edema and behavioral changes in rats. Contraception 1984; 29:283-9.

10.Olivennes F, Fanchin R, Bouchard P, Taïeb J, Selva J, Frydman R. Scheduled administration of a gonadotrophin-releasing hormone antagonist (Cetrorelix) on day 8 of in-vitro fertilization cycles: a pilot study. Hum Reprod 1995; 10:1382-6.

11. Halmos G, Schally AV, Pinski J, Vadillo-Buenfil M, Groot K. Down-regulation of pituitary receptors for luteinizing hormone-releasing hormone (LH-RH) in rats by LH-RH antagonist Cetrorelix. Proc Natl Acad Sci USA 1996; 93:2398-402.

12. Olivennes F, Fanchin R, Bouchard P, et al. The single or dual administration of the gonadotrophinreleasing hormone antagonist Cetrorelix in an in vitro fertilization-embryo transfer program. Fertil Steril 1994; 62:468-76.

13. Olivennes F, Diedrich K, Frydman R, et al. Safety and efficacy of a $3 \mathrm{mg}$ dose of the GnRH antagonist cetrorelix in preventing premature LH surges: report of two large multicentre, multinational, phase IIIb clinical experiences. Reprod Biomed Online 2003; 6:432-8.

14.Al-Inany $\mathrm{H}$, Aboulghar $\mathrm{M}$. GnRH antagonist in assisted reproduction: a Cochrane review. Hum Reprod 2002; 17:874-85.

15. Hernandez ER. Embryo implantation and GnRH antagonists: embryo implantation: the Rubicon for GnRH antagonists. Hum Reprod 2000; 15:1211-6.

16.Engel JB, Ludwig M, Junge K, Howles CM, Diedrich $K$. No influence of body weight on pregnancy rate in patients treated with cetrorelix according to the single- and multiple-dose protocols. Reprod Biomed Online 2003; 6:482-7.

17. Shapiro DB. GnRH antagonists in normal responder patients. Fertil Steril 2003; 80 (Suppl 1):S8-15.

\section{Conflitos de Interesses:}

O Laboratório Asta Médica pela cessão dos medicamentos utilizados neste estudo.

Recebido em: 12/9/2003

Aceito com modificações em: 6/10/2003 